\title{
Emergence And Management Of Muti-Drug Resistant Pseudomonas Aeruginosa Isolated From Intensive-Care Unit
}

Saman Nadeem, Faisal Hanif, Yasmeen Taj, Sana Yousuf, Nadia Midhat Zehra, Pervaiz Asghar

- - - - - - - - - - - - - - - - - - - - - - - - - - - - ABSTRACT

Objective: To evaluate the antibiotic resistance pattern of Pseudomonas aeruginosa and its prevalence from samples received from Intensive Care Units (adult and neonatal) at PNS Shifa Hospital Karachi.

Study Design and Setting: This cross sectional study was carried out at PNS SHIFA hospital.

Methodology: Samples including blood, pus, wound swab, sputum and endobronchial washing were received for culture and sensitivity. Isolates were cultured on blood and MacConkey agar. Antibiotic susceptibility testing was performed by Kirby-Bauer and broth microdilution and then analyzed on SPSS version 23.Results were confirmed by VITEK 2.

Results: Out of 674 positive clinical specimens 97(14.39\%) were positive for Pseudomonas aeruginosa growth. The most susceptible antibiotic against Pseudomonas aeruginosa was Polymixin b (94.854\%). The least effective antimicrobial was aztreonam (40.21\%sensitive).

Conclusion: The prevalence of Pseudomonas aeruginosa from the samples of Intensive Care Units was found to be $14.39 \%$. The most susceptible antibiotic against pseudomonas was Polymixin B. The least effective antimicrobial was aztreonam.

Keywords: Antibiotic resistance pattern, Culture and sensitivity, Pseudomonas aeruginosa.

\section{INTRODUCTION:}

Pseudomonas aeruginosa is an opportunistic pathogen causing a wide range of infections in the human host. ${ }^{1,2}$ It is saprophytic in nature and is found in both hospital and community settings.It can survive in adverse conditions because of its extremely flexible physiological and physical nature. ${ }^{2}$ Pseudomonas aeruginosa is one of the most important pathogen causing soft tissue infections in burns, diabetic patients and the immunocompromised host. Although not a human flora it usually causes super-infection in diabetic, burns and cystic fibrosis patients. ${ }^{3}$

About $9 \%$ of all healthcare associated infections are caused by Pseudomonas aeruginosa. ${ }^{4}$ Pathogenicity of organism

Saman Nadeem,
Registrar, Department of Pathology
Bahria University Medical \& Dental College / PNS SHIFA Hospital
Earachi
Faisal Hanif,
Assistant Professor, Department of Pathology
Bahria University Medical \& Dental College/ PNS SHIFA Hospital
Karachi
Yasmeen Taj,
Professor, Department of Pathology
| Bahria University Medical and Dental College
Sana Yousuf,
Begistrar, Department of Pathology
| Karachi University Medical \& Dental College / PNS SHIFA Hospital
| Nadia Midhat Zehra,
| Registrar, Department of Pathology
| Karia University Medical \& Dental College / PNS SHIFA Hospital
| Pervaiz Asghar
| Bahria University Medical and Dental College
| Accepted: 13-02-19 is directly linked to the toxins and enzymes produced by it, and its ability to resist phagocytosic immune capacity of the host. ${ }^{3,4}$ Emerging antibiotic resistance against different antimicrobials contributes to its increased morbidity and mortality. Formation of biofilms, increased drug permeability, multi-drug efflux mechanism and acquisition of certain enzymes are the factors mainly responsible for acquisition of antibiotic resistance. ${ }^{1-4}$

According to records from the Centre of Disease Control and National Nosocomial Infection Surveillance System (NNIS) Pseudomonas aeruginosa is the second principal cause of pneumonia (17\%), third cause of UTI (7\%)fourth cause of surgical wound infection(8\%) and the seventh leading cause of sepsis $(2 \%)$ in the hospital settings. Pseudomonas aeruginosa is one the most common cause of health care associated infections specifically from intensive care units.Multidrug resitant strains and carabapenem resistant isolates have been isolated from intensive care units with limited treatment options available. ${ }^{5}$

Emerging resistant strains of pseudomonas have strongly restricted the treatment possibilities available. This study was aimed to evaluate the antibiotic resistance pattern of Pseudomonas aeruginosa and its prevalence from samples received from Intensive Care Units (adult and neonatal) at PNS Shifa Hospital Karachi.

\section{METHODOLOGY:}

The cross sectional study was performed from January 2016 to June2017 on patients admitted to the Intensive Care Units (both adult and neonatal) in PNS Shifa hospital. After approval of ethical committee of the hospital we included samples received from patients admitted to the neonatal and adult intensive care units. They include body fluids, blood, 
and pus,endobronchial washing, sputum, collected and submitted to the microbiology department for culture and sensitivity.

Samples received from different body sites were inoculated on Blood, MacConkey and Chocolate agar and incubated at $37 \mathrm{C}$ for 24 hours both aerobically and anaerobically. Standard biological methods were carried out in order to identify Pseudomonas aeruginosa colonies as outlined by CLSI. Biochemical test like oxidase, urease, citrate utilization test, nitrate reduction and indole production were also performed. Confirmation of Pseudomonas aeruginosa was done by using API 20NE (Biomeriux).

Antimicrobial susceptibility testing was carried out by Kirbybaeur disc diffusion method and broth microdilution as outlined by clinical laboratory standard international guidelines. ${ }^{6}$ Isolated colonies of Pseudomonas aeruginosa were picked up by a sterile loop and $0.5 \mathrm{McF}$ arland suspension was prepared. It was then inoculated on the surface of MuellerHinton agar (MHA) plate using sterile cotton swab and incubated at $37 \mathrm{C}$ for 24 hours after placing different antibiotic discs.

Eleven antibiotic discs used in this study were Polymixin $\mathrm{b}$ (Oxoid) (300 units), imipenem (Oxoid) (10 $\mu \mathrm{g})$ tazobactampiperacillin (Oxoid) $(110 \mu \mathrm{g})$, aztreonam (Oxoid) $(30 \mu \mathrm{g})$ ceftazidime (Oxoid) $(30 \mu \mathrm{g})$ ceftazidime (Oxoid) $(30 \mu \mathrm{g})$,

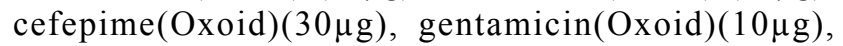

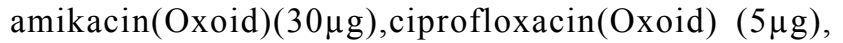
tazobactam/piperacillin(Oxoid)(110 $\mu \mathrm{g})$, cefoperazone/ sulbactam $75 / 10 \mathrm{mcg})$, meropenem(Oxoid)(10 $\mu \mathrm{g})$, imipenem (Oxoid) $(10 \mu \mathrm{g})$, aztreonam (Oxoid) $(30 \mu \mathrm{g})$. The results were interpreted as per CLSI guidelines. ${ }^{8}$ Control strains used were Escherichia coli ATCC 25922 and Pseudomonas aeruginosa ATCC 27853.

All isolates identification and antimicrobial susceptibility patters has been confirmed by VITEK 2(bioMérieux).

Data was analyzed using statistical software SPSS Version 23. $P$ value of less than 0.05 was considered significant.

\section{RESULTS:}

In this study 97 (14.39\%) out of 674 samples were positive for the growth of Pseudomonas aeruginosa. Clinical specimens showing positive growth for Pseudomonas aeruginosa include pus (49\%),blood (25\%), sputum (13\%), endobronchial washing (13\%) (Figure: 1). Among them $33 \%$ of samples were received by females and $67 \%$ by males. The results of antimicrobial susceptibility testing is shown in (Figure:2 ). The most susceptible antibiotic against pseudomonas is Polymixin B $(94.85 \%)$ sensitive followed by gentamicin $(89.69 \%)$ and amikacin(84.54\%) imipinem (77.32\%) cefoperazone/salbactum(75.26), meropenem (71.13\%), cefepime(68.04\%) ciprofloxacin(56.7\%). The least effective antimicrobial is aztreonam ( $40.21 \%$ sensitive $)$ followed by ceftazidime( $56.7 \%$ ) sensitive.

\section{DISCUSSION:}

Pseudomonas aeruginosa is one of the most important opportunistic pathogen. It has imposed a huge burden on healthcare with increase in mortalities both in hospitals and community settings. ${ }^{1-4}$. In our study, $67 \%$ isolates were from males and $33 \%$ from females which is comparable to a study carried out in Baroda ${ }^{1}$, however these figures determined are in disagreement with the values observed in Nepal where female prevelance is superceding in Pseudomonas Spp. ${ }^{7}$ This change may be due to the difference in cultural practices.

Most of samples showing the growth of Pseudomonas aeruginosa (49\%) were from pus followed by blood(29\%) in intensive care unit. These results are in slight variation

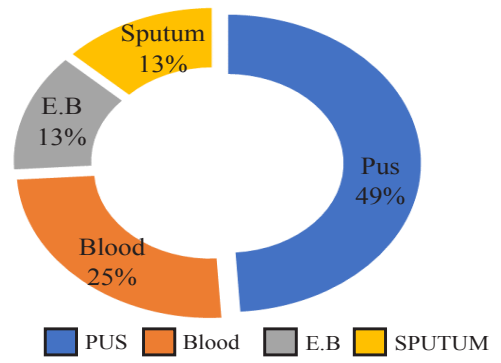

Figure 1 Sample wise prevalence of pseudomonas isolates

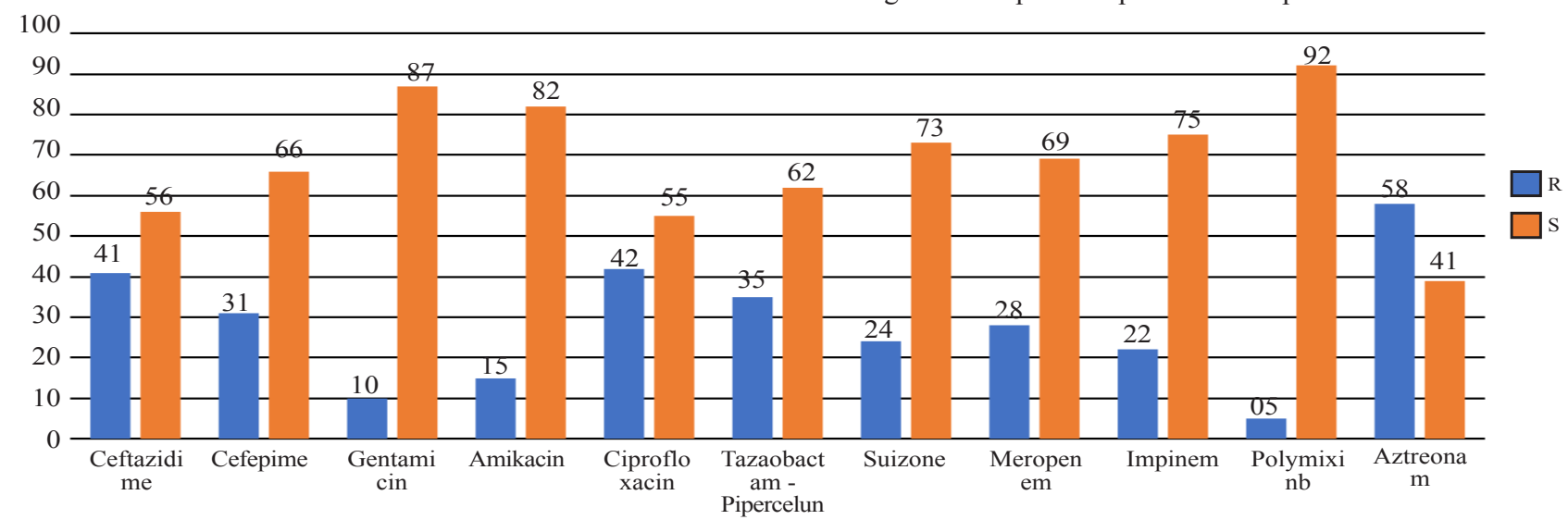

Figure 2:Antimicrobial susceptibility pattern of pseudomonas aeruginosa isolates 
with a study carried out in Baroda, revealed Pseudomonas growth $(70 \%)$ from wound swab. ${ }^{1}$ Although in our setup, pus samples revealed the growth of Pseudomonas as wound swabs are not an appropriate sample for culture and sensitivity.Pseudomonal infections are difficult to treat as the organism exhibit intrinsic resistance towards many antimicrobials. Microorganisms have developed numerous mechanisms to counter the effect of antimicrobials making them resitant. The antibiotic susceptibility differs from hospital to hospital,of different regions and may also alter with time. Patients came in contact with various antibiotics in rural or urban areas in sub-optimal doses and exploitation of some over the counter drugs are the contributing factors towards emerging resistance. Pseudomonas aeruginosa resistance to antimicrobials is either through acquiring carbapenemases or aminoglycoside-modifying enzymes. ${ }^{2,45}$ Resistance is conferred by the shift of extrachromosomal DNA between different isolated generating a population of bacteria that becomes more difficult to treat. In our study, the most effective antibiotic found was Polymixin $b$. This finding may be due to the judicial use of this antibiotic to treat more resistant isolates, as it is the last resort for the clinicians to treat such resistant infections. Moreover Polymixin $b$ is not available in oral form in Pakistan and it has major side effects like nephrotoxicity which is also one of the reasons of its limited use and poor patient compliance. These can be the causes contributing towards its highest sensitivity observed against pseudomonal isolates in our study. Quinolones are one of the best treatment options for UTI mainly because of the easy accessibility in oral formula,good patient compliance due to its pharmacokinetics and cheaper cost. However, emerging quinolone resistant strains of Pseudomonas aeruginosa have been reported due to the changes in target sites of the bacteria and active efflux pumps operated to prevent the entry of the drugs. ${ }^{8,9}$ These are the common factors contributing towards increasing resistance against ciprofloxacin. In our study resistance against ciprofloxacin is $43.3 \%$ which is quite similar to the figures reported in one of their studies carried in India which revealed the resistance rate of $31.5 \%$.Resistance shown by these isolates are either acquired or due to the overexpression of MexABOprM efflux pump. ${ }^{10}$ One of the recent studies carried out in Malaysia reported a high resistance pattern of ciprofloxacin against pseudomonas $(92 \%) .{ }^{11}$ Slight discrepancy observed between the results might be due to the patient awareness and clinician's approach to treat the patients admitted in intensive care units .

One striking observation in our study is the rising trend of resistance of pseudomonal isolates against carbapenems (meropenem 28.87\% resistantt and imipinem 22.86\% resistant.The figures observed are quite identical with another research conducted in Pakistan in 2018 showing a huge reistance rate due to the production of blaVIM Metallo- â-lactamase. ${ }^{12}$ Carbapenem-resistant strains of Pseudomonas aeruginosa have been reported in various countries like Korea and China. ${ }^{13,14}$ These strains resist carbapenems ( both Imipenem and Meropenem). Regular use of Imipenem alters the outer membrane absorbency or when given along with Piperacillin, changes the target site of Pseudomonas aeruginosa which then eventually turned them resistant to carbapenems.

Results obtained for resistance towards the antibiotic amikacin(15.46\%). are also in accordance with a study conducted in Pakistan in 2017 in Peshawar showing amikacin the most sensitive antimicrobial against pseudomonas isolates. ${ }^{15}$ Higher resistance against aminoglycosides are also reported in studies carried out in Bangladesh and India. ${ }^{16}$ Good sensitivity pattern is observed with the antimicrobials cefoperazone-salbactum (75.26\%) which is in close similarity with a study conducted in China showing cefoperazonesulbactam and amikacin are the choice of drugs available for carbapenem resistant strains, although their combination have no added advantage. ${ }^{17}$ Cefoperazone acts on the cell wall, and salbactum acts as a beta-lactamase inhibitor, to amplify the bactericidal activity of cefoperazone against beta-lactamase-producing isolates.

The most resistant antibiotic found in our study is aztreonam $59.79 \%$. Same resistance pattern was also reported in Saudi Arabia (63.4\%). ${ }^{18}$ Aztreonam has excellent efficacy against Gram-negative aerobic bacteria. Aztreonam has found to be more active than cefamandole in certain infections and quite same in efficacy to tobramycin or gentamicin. ${ }^{19}$ Aztreonam has been proven to be an effective antibiotic in treating pseudomonal infections in most patients including burns and soft tissue infections (except in cystic fibrosis). ${ }^{20}$ However aztreonam reistance has been emerging due to variable genetic mutations in pseudomonal isolates. ${ }^{21}$ Combination therapies like aztreonam-monobactam can be a good option available for such resitant strains, but still under clinical trials for the treatment of serious infections caused by metallo-beta lactamase producing pseudomonas. ${ }^{22}$ Therefore the inadequate number of pseudomonal infections available for our study from intensive care units thwarts any conclusions as to the relative efficacy of aztreonam compared with other antibiotic options available.

The antibiotic susceptibility pattern observed thin down the most sensitive drugs from the most resistant ones and our study can be used to provide a more detailed and comprehensive attitude to opt the most appropriate antibiotic therapy against Pseudomonas aeruginosa. Aztreonam, is an antibiotic used primarily to treat infections caused by Pseudomonas aeruginosa. The high resistance pattern observed in our study is quite alarming for both clinicains and microbiologist.We suggest a judicial and restricted use of this antibiotic in hospital settings. Regular monitoring of antibiotics with proper de-escalation should be adopted in order to decrease the emergence of such resistant strains. 


\section{CONCLUSION:}

The prevelance of Pseudomonas aeuginosa isolated from the samples received at intensive care units(adult and neonatal) was $14.39 \%$. Polymixin B was the only antimicrobial showing highest susceptibility trends against Pseudomonal isolates. Piperacillin-tazobactam is the treatment of choice for non-resistant isolates. Aminoglycosides like amikacin and gentamicin can also be one of treatment options available but cannot be used alone due to their poor intracellular penetration.

\section{REFERENCES:}

1. Patel H, Garala RN. Antibiotic susceptibility pattern of Pseudomonas aeruginosa isolated at SSG hospital Baroda. J Res Med Den Sci 2014;2:84-7.

2. Rouhi S, Ramazanzadeh R. Prevalence of bla (Oxacillinase23)and bla(Oxacillinase-24/40-)type Carbapenemases in Pseudomonas aeruginosa Species Isolated From Patients With Nosocomial and Non-nosocomial Infections in the West of Iran. Iran J Pathol. 2018;13(3):348-356.

3. Sekhar M S, M K U, Rodrigues GS, Vyas N, Mukhopadhyay C. Antimicrobial susceptibility pattern of aerobes in diabetic foot ulcers in a South-Indian tertiary care hospital. Foot (Edinb) 2018;37:95-100.

4. Yusuf E, Van HB, Verbrugghe W, Leven M, Goovaerts E, Bergs K, et al. Emergence of antimicrobial resistance to Pseudomonas aeruginosa in the intensive care unit: association with the duration of antibiotic exposure and mode of administration. Ann Intensive Care. 2017;7(1):72.

5. Maraolo AE, Cascella M, Corcione S, Cuomo A, Nappa S, Borgia G, De Rosa FG, Gentile I.Management of multidrugresistant Pseudomonas aeruginosa in theintensive care unit: state of the art.Expert Rev Anti Infect Ther.2017;15(9):861-71.

6. Clinical and Laboratory Standards institute (CLSI). Performance Standards for Antimicrobial Susceptibility Testing. 27th ed. Wayne, PA: 2017.

7. Acharya M, Joshi PR, Thapa K, Aryal R, Kakshapati T, Sharma S. Detection of metallo-â-lactamases-encoding genes among clinical isolates of Pseudomonas aeruginosa in a tertiary care hospital, Kathmandu, Nepal. BMC Res Notes. 2017;10(1):718.

8. Kos VN, Déraspe M, McLaughlin RE, Whiteaker JD, Roy $\mathrm{PH}$, Alm RA, Corbeil J, Gardner H. The resistome of Pseudomonas aeruginosa in relationship to phenotypic susceptibility. Antimicrob Agents Chemother. 2015;59(1):42736.

9. Abbara S, Pitsch A, Jochmans S, Hodjat K, Cherrier DP, Monchi M, Vinsonneau C, Diamantis S. Impact of a multimodal strategy combining a new standard of care and restriction of carbapenems, fluoroquinolones and cephalosporins, on antibiotic consumption and resistance of P.aeruginosa in an ICU. Int $J$ Antimicrob Agents. 2018;(18)30361-3.

10. Choudhury D, Ghose A, Dhar Chanda D, Das Talukdar A, Dutta Choudhury M, PaulD, Maurya AP, Chakravarty A, Bhattacharjee A. Premature Termination of MexR Lead to Overexpression of MexAB-OprM Efflux Pump in Pseudomonas aeruginosa in aTertiary Referral Hospital in India. PLoS One. 2016 ;11(2):e0149-156.
11. Al-Kabsi AM, Yusof MYBM, Sekaran SD. Antimicrobial resistance pattern of clinical isolates of Pseudomonas aeruginosa in the University of Malaya Medical Center, Malaysia. Afr J Microbiol Res 2011;5(29):5266-72.

12. Qureshi R, Shafique M, Shahid M, Rasool MH, Muzammil S. Molecular detection of blaVIM Metallo-â-lactamase producing clinically isolated Pseudomonas aeruginosa from tertiary care hospital, Faisalabad. Pak J Pharm Sci. 2018;31:2673-77.

13. Cho HH, Kwon GC, Kim S, Koo SH. Distribution of Pseudomonas-Derived Cephalosporinase and Metallo-âLactamases in Carbapenem-Resistant Pseudomonas aeruginosa Isolates from Korea. J Microbiol Biotechnol. 2015;25(7):1154-62.

14. Yin D, Wu S, Yang Y, Shi Q, Dong D, Zhu D, Hu F. In Vitro Activity of Ceftazidime-Avibactam and CeftolozaneTazobactam against Clinical Isolates of Enterobacteriaceae and Pseudomonas aeruginosa: Results from a Multicenter Studym in China, 2017. Antimicrob Agents Chemother. 2019 Jan 7. pii: AAC.02431-18.

15. Samad A, Ahmed T, Rahim A, Khalil A, Ali I. Antimicrobial susceptibility patterns of clinical isolates of Pseudomonas aeruginosa isolated from patients o respiratory tract infections in a Tertiary Care Hospital, Peshawar. Pak J Med Sci. 2017;33(3):670-674.

16. Rashid A, Chowdhury A, Rahman SHZ, Begum SA, Muazzam $\mathrm{N}$. Infections by Pseudomonas aeruginosa and antibiotic resistance pattern of the isolates from Dhaka Medical College Hospital. Bangladesh J Med Microbiol 2007;1(2):48-51.

17. Cai Y, Yang D, Wang J, Wang R. Amikacin and cefoperazone / sulbactam alone or incombination against carbapenemresistant Pseudomonas aeruginosa. Diagn Microbiol Infect Dis. 2018;91(2):186-190.

18. Ahmad S, Harbi MNA. Antibiotic susceptibility pattern of isolates of Pseudomonas aeruginosa in a Saudi Arabian Hospital. BJMS 2014; 13:45-8.

19. Yamagishi Y, Hagihara M, Kato H, Hirai J, Nishiyama N, Koizumi Y, Sakanashi D,Suematsu H, Nakai H, Mikamo H. In vitro and in vivo Pharmacodynamics of Colistin and Aztreonam Alone and in Combination against MultidrugResistant Pseudomonas aeruginosa. Chemotherapy. 2017;62(2):105-110.

20. Radan M, Moniri R, Khorshidi A, Gilasi H, Norouzi Z, Beigi F, Dasteh Goli Y.Emerging Carbapenem-Resistant Pseudomonas aeruginosa Isolates Carrying bla(IMP)Among Burn Patients in Isfahan, Iran. Arch Trauma Res. 2016;5(3):e33664.

21. Jorth P, McLean K, Ratjen A, Secor PR, Bautista GE, Ravishankar S, Rezayat A, Garudathri J, Harrison JJ, Harwood RA, Penewit K, Waalkes A, Singh PK, Salipante SJ. Evolved Aztreonam Resistance Is Multifactorial and Can Produce Hypervirulence in Pseudomonas aeruginosa. MBio. 2017 ;8(5). pii: e00517-17.

22. Karlowsky JA, Kazmierczak KM, de Jonge BLM, Hackel MA, Sahm DF, Bradford PA. In Vitro Activity of AztreonamAvibactam against Enterobacteriaceae and Pseudomonas aeruginosa Isolated by Clinical Laboratories in 40 Countries from 2012 to 2015. Antimicrob Agents Chemother. 2017;61(9). pii: e00472-17. 